\title{
Polissacarídeo de Escherichia coli na Prevenção da Infeção do Trato Urinário Recorrente: Uma Revisão Baseada na Evidência
}

\section{Polysaccharide of Escherichia coli in the Prevention of Recurrent Urinary Tract Infection: An Evidence-Based Review}

\author{
Manuel TINOCO ${ }^{1}$, Mélody SANTOS ${ }^{1}$, Conceição REITOR ${ }^{1}$, Andreia RODRIGUES ${ }^{1}$, João MONTEIRO ${ }^{1}$ \\ Acta Med Port 2018 Mar;31(3):165-169 - https://doi.org/10.20344/amp.9367
}

\section{RESUMO}

Introdução: A infeção do trato urinário recorrente é frequente e associa-se a morbilidade, custos e aquisição de resistências a antibióticos. A vacina OM 8930 é constituída por um polissacarídeo de Escherichia coli e é uma possível medida profilática, mas existe dúvida quanto à sua efetividade.

Material e Métodos: Realizámos uma revisão sistemática das principais bases de dados de medicina baseada na evidência, com seleção criteriosa dos artigos encontrados.

Resultados: Verificámos uma efetiva diminuição do número de recorrências nos grupos experimentais.

Discussão: Admitimos a recomendação do uso da vacina como profilaxia da cistite recorrente.

Conclusão: A vacina tem impacto na recidiva da cistite recorrente. Sugerimos a realização de mais estudos para avaliar o custo/ benefício da vacina, bem como o seu efeito nas infeções urinárias complicadas.

Palavras-chave: Adjuvantes Imunológicos/uso terapêutico; Escherichia coli Uropatogénica; Infecções por Escherichia coli; Infecções Urinárias

\section{ABSTRACT}

Introduction: Recurrent urinary tract infection is frequent and it is related to morbidity, costs and growing antibiotic resistance. OM 8930 vaccine is composed by Escherichia coli polysaccharide and it is a possible prophylactic measure, but there is doubt as to its effectiveness.

Material and Methods: We performed a systematic review in evidence-based medicine databases with rigorous bibliography selection. Results: We found an effective decrease of recurrences in test groups.

Discussion: We recommend the administration of the vaccine as prophylaxis of recurrent cystitis.

Conclusion: The vaccine has impact on the recurrent cystitis relapse rate. We suggest that more studies be carried out to evaluate the vaccine cost-benefit and its effectiveness in complicated urinary infections.

Keywords: Adjuvants, Immunologic/therapeutic use; Escherichia coli Infections; Urinary Tract Infections; Uropathogenic Escherichia coli

\section{INTRODUÇÃO}

A infeção do trato urinário (ITU) é frequente em cuidados de saúde primários e tem morbilidade e custos associados. ${ }^{1} \mathrm{~A}$ cistite aguda não complicada é o tipo de ITU mais frequente e é causada principalmente pela Escherichia coli (E. coli) ${ }^{1}$

A ITU recorrente é definida pela presença de pelo menos dois episódios de ITU em seis meses ou de pelo menos três episódios em 12 meses. ${ }^{2}$ Nas mulheres com idade entre os 18 e 39 anos, a prevalência de recorrência aos seis meses é de $24 \%$.

Das medidas não antimicrobianas para a profilaxia da ITU recorrente, destaca-se a vacina imunoestimulante oral - OM 8930 / Uro-Vaxom ${ }^{\circledast}$ - a única existente, sendo constituída por lisado de polissacarídeos de $E$. coli. ${ }^{1,2} \mathrm{~A}$ opção pela imunoprofilaxia em vez da profilaxia antibiótica poderá evitar o aumento das resistências aos antimicrobianos pela menor utilização dos mesmos. ${ }^{1}$

Em Portugal, o tratamento custa aproximadamente $€ 59$, não é comparticipado, e há dúvidas quanto à sua eficácia.

Neste contexto foi elaborada uma revisão baseada na evidência, com o objetivo de determinar a eficácia qualitativa da vacina na prevenção da recorrência da ITU.

\section{MATERIAL E MÉTODOS}

Efetuámos uma pesquisa, em abril de 2016, na Bandolier, Cochrane Library, DARE, Canadian Medical Association, National Guideline Clearinghouse e NICE. O Índex de Revistas Médicas Portuguesas também foi consultado.

Utilizámos os descritores Medical Subject Headings (MeSH) urinary tract infections e Uro-Vaxom ${ }^{\circledast}$ (equivalente de 'OM 8930').

Os critérios de inclusão utilizados foram:

- população: adultos com ITU recorrente;

- intervenção: utilização de polissacarídeo de E. coli;

- comparação: placebo ou pré-tratamento;

- outcome: diminuição da recorrência da ITU.

1. Unidade Saúde Familiar Casa dos Pescadores. Póvoa de Varzim. Portugal.

$\triangle$ Autor correspondente: Manuel Tinoco. manel_tinoco@hotmail.com

Recebido: 29 de junho de 2017 - Aceite: 12 de fevereiro de 2018 | Copyright @ Ordem dos Médicos 2018 
Definimos como critérios de exclusão os estudos com população infantil, juvenil ou animal, estudos moleculares ou artigos redigidos em idioma não dominado pelos autores.

Utilizámos a escala Strength Of Recommendation Taxonomy da American Academy of Family Physicians ${ }^{3}$ para a classificação dos níveis de evidência e a atribuição da força de recomendação.

\section{RESULTADOS}

Do total de 97 resultados encontrados, 54 foram excluídos por não cumprirem os critérios de inclusão, 21 por repetição, 13 pela população / estudos moleculares, e dois por serem redigidos em russo. Assim, sete cumpriram todas as condições: dois ensaios clínicos aleatorizados, uma coorte prospetiva, duas metanálises e duas guidelines; estes datam de janeiro de 1990 até março de 2015 (Tabela 1).

\section{Ensaios clínicos aleatorizados}

Um dos ensaios clínicos, Tammen et al, ${ }^{4}$ duplamente cego, contemplou uma amostra de 120 participantes com ITU recorrente, aos quais foi administrado durante três meses, diariamente, uma cápsula de polissacarídeo de E. coli no grupo experimental, ou placebo no grupo controle; foram monitorizados durante mais três meses. Verificou-se uma diminuição do número de recorrências de ITU $(p<0,01)$, da bacteriúria $(p<0,01)$ e da disúria $(p<0,05)$ no grupo experimental. Dos 120 participantes, 57 foram seguidos por mais cinco meses, verificando-se menos recorrências no grupo experimental $(p<0,05)$.

O outro ensaio clínico, Magasi et al, ${ }^{5}$ também duplamente cego, contemplou uma amostra de 112 participantes

Tabela 1 - Descrição dos dois ensaios clínicos aleatorizados selecionados na pesquisa

\begin{tabular}{|c|c|c|}
\hline Referência & Tammen $\mathrm{H}$ et $a l^{4}$ & Magasi et a/ ${ }^{5}$ \\
\hline $\begin{array}{l}\text { Critérios de } \\
\text { inclusão }\end{array}$ & $\begin{array}{l}\geq 2 \text { infeções do trato urinário nos } 6 \text { meses prévios } \\
\text { ao ensaio e com agudização no início do mesmo ( } \geq \\
10^{5} \text { microrganismos } / \mathrm{mL} \text { na amostra de urina de jato } \\
\text { médio ou } \geq 10^{4} \text { na amostra por cateter). }\end{array}$ & $\begin{array}{l}\text { ITU recorrente, com bacteriúria no início do estudo ( } \geq 10^{5} \\
\text { microrganismos/ml em amostra de urina de jato médio). } \\
\text { Pressupomos existência de agudização no início do } \\
\text { estudo, uma vez terem realizado ciclo de antibioterapia. }\end{array}$ \\
\hline $\begin{array}{l}\text { Critérios de } \\
\text { exclusão }\end{array}$ & $\begin{array}{l}\text { disúria na ausência de bacteriúria } \\
\text { algaliação } \\
\text { gravidez } \\
\text { cistite recorrente pós-coital } \\
\text { anomalias do trato urinário }\end{array}$ & $\begin{array}{l}\text { uropatia obstrutiva } \\
\text { pielonefrite crónica } \\
\text { algaliação } \\
\text { refluxo vesico-ureteral } \\
\text { urolitíase }\end{array}$ \\
\hline População & $\begin{array}{l}150 \text { participantes } \\
\text { excluídos } 30 \text { da análise estatística } \\
80 \% \text { follow-up completo } \\
\text { idade: } 48 \text { - } 53 \text { anos } \\
\text { ambos os sexos ( } 14,2 \% \text { homens) } \\
\text { grupos homogéneos na distribuição dos fatores } \\
\text { idade, sexo e antecedentes clínicos }\end{array}$ & $\begin{array}{l}122 \text { participantes } \\
\text { excluídos } 10 \text { da análise estatística } \\
91,8 \% \text { follow-up completo } \\
\text { idade: } 18 \text { - } 82 \text { anos } \\
\text { ambos os sexos ( } 15,2 \% \text { homens) } \\
\text { grupos homogéneos na distribuição dos fatores idade, } \\
\text { sexo e antecedentes clínicos }\end{array}$ \\
\hline Intervenção & $\begin{array}{l}1^{a} \text { etapa: ciclo de antibioterapia. } \\
2^{a} \text { etapa: } 3 \text { meses de tratamento com } 6 \text { mg/dia de } \\
\text { Polissacarídeo de Escherichia coli ou placebo; } \\
3^{\text {a }} \text { etapa: } 3 \text { meses de observação; } \\
57 \text { indivíduos foram ainda observados por mais } 5 \\
\text { meses; } \\
\text { antibioterapia administrada se infeção no período } \\
\text { dos } 6 \text { meses. }\end{array}$ & $\begin{array}{l}1^{\text {a }} \text { etapa: ciclo de antibioterapia; } \\
2^{\text {a }} \text { etapa: } 3 \text { meses de tratamento com } 6 \text { mg/dia de } \\
\text { Polissacarídeo de Escherichia coli ou placebo; } \\
3^{\text {a }} \text { etapa: } 3 \text { meses de observação; } \\
\text { antibioterapia administrada se infeção no período dos } 6 \\
\text { meses. }\end{array}$ \\
\hline Outcome & $\begin{array}{l}\text { Frequência das infeções do trato urinário: } \\
\text { bacteriúria (definida como } 10^{4} \text { microrganismos } / \mathrm{mL} \text { ) } \\
\text { e sintomas urinários; }\end{array}$ & $\begin{array}{l}\text { Frequência das infeções do trato urinário: bacteriúria } \\
\text { (com } 10^{5} \text { microrganismos } / \mathrm{mL} \text { ) e sintomas urinários. }\end{array}$ \\
\hline $\begin{array}{l}\text { Resultados } \\
\text { (grupo } \\
\text { experimental) }\end{array}$ & $\begin{array}{l}\text { Diminuição do número de recorrências de infeções } \\
\text { do trato urinário, quer durante os } 3 \text { meses de } \\
\text { tratamento }(p<0,05) \text {, quer nos } 3 \text { meses seguintes } \\
\text { de observação }(p<0,01) ; p<0,001 \text { no global dos } \\
6 \text { meses e } p<0,05 \text { no grupo de follow-up adicional } \\
\text { de } 5 \text { meses; } \\
\text { diminuição da bacteriúria }(p<0,01) \text {, disúria } \\
(p<0,05) \text { e nitritúria }(p<0,05) \text {; } \\
\text { diminuição da leucocitúria. }\end{array}$ & $\begin{array}{l}\text { Diminuição do número de recorrências de ITU ao fim de } 6 \\
\text { meses }(p<0,0005) \text {; } \\
\text { diminuição da bacteriúria ( } p<0,001) \text {, disúria }(p<0,005) \text { e } \\
\text { leucocitúria }(p<0,005) \text { ao fim dos } 6 \text { meses; } \\
67,2 \% \text { dos doentes tratados não tiveram recorrências } \\
(p<0,0005) \text {. }\end{array}$ \\
\hline $\begin{array}{l}\text { Nível de } \\
\text { evidência }\end{array}$ & 1 & 1 \\
\hline
\end{tabular}


com ITU recorrente, durante um período de tratamento e monitorização semelhantes ao estudo anterior. No final, verificou-se uma diminuição da recorrência de ITU no grupo experimental $(p<0,0005)$. Do total dos 112 participantes,
$67,2 \%$ não teve recorrência de ITU ( $p<0,0005)$. Verificou-se ainda uma redução da bacteriúria, disúria e leucocitúria no grupo experimental, aos três meses e no final $(p<$ 0,0005 e $p<0,001$, respetivamente) (Tabela 2).

Tabela 2 - Descrição da coorte selecionada na pesquisa

\begin{tabular}{|c|c|}
\hline Referência & Kim et $a l^{6}$ \\
\hline Critérios de inclusão & $\begin{array}{l}\text { ITU recorrente ( } \geq 2 \text { episódios de cistite, nos } 6 \text { meses que antecederam o estudo, definida como } \geq 10^{5} \\
\text { microrganismo } / \mathrm{mL} \text { e } \geq 6 \text { leucócitos/ campo em amostra de urina), em agudização no início do estudo. }\end{array}$ \\
\hline Critérios de exclusão & $\begin{array}{l}\text { refluxo vesicouretral } \\
\text { uropatia obstrutiva } \\
\text { litíase urinária } \\
\text { insuficiência renal } \\
\text { intervenções no trato urinário }\end{array}$ \\
\hline População & $\begin{array}{l}42 \text { participantes; } 8 \text { excluídos da análise estatística } \\
80,9 \% \text { de follow-up completo } \\
\text { idade: } 34 \text { - } 75 \text { anos; apenas mulheres }\end{array}$ \\
\hline Intervenção & $\begin{array}{l}1^{\text {a }} \text { etapa: ciclo de antibioterapia para tratamento da ITU inicial; } \\
2^{\mathrm{a}} \text { etapa: } 3 \text { meses de tratamento com polissacarídeo de Escherichia coli, } 6 \mathrm{mg} / \text { dia; foram excluídos os } \\
\text { indivíduos onde ocorreu agudização nesta fase; } \\
3^{\mathrm{a}} \text { etapa: observação durante } 6 \text { meses. } \\
\text { antibioterapia administrada se infeção no período dos } 6 \text { meses de observação. }\end{array}$ \\
\hline Outcome & $\begin{array}{l}\text { Número de recorrências de cistite após a imunoprofilaxia; } \\
\text { gravidade dos sintomas (com utilização de escala validada). }\end{array}$ \\
\hline Resultados & $\begin{array}{l}\text { Comparação do número de episódios de ITU entre os } 6 \text { meses pré e pós tratamento com } \\
\text { polissacarídeo de Escherichia coli: } \\
28 \text { indivíduos sem recorrência em } 6 \text { meses }(p<0,001) \text {; } \\
\text { nos } 6 \text { indivíduos em que a infeção recorreu, a gravidade dos sintomas foi menor; } \\
\text { a vacina diminuiu } 3,9 \text { vezes as agudizações de infeção urinária recorrente, e também a gravidade dos } \\
\text { sintomas na agudização. }\end{array}$ \\
\hline
\end{tabular}

Nível de evidência

1

ITU: infeção do trato urinário

Tabela 3 - Descrição das duas metanálises selecionadas na pesquisa

\begin{tabular}{|c|c|c|}
\hline Referência & Bauer et $\mathrm{al}^{7}$ & Naber et al ${ }^{8}$ \\
\hline População & $\begin{array}{l}5 \text { ensaios: } 108 \text { a } 171 \text { participantes em cada } \\
<20 \% \text { homens em cada }\end{array}$ & $\begin{array}{l}4 \text { ensaios: } 122 \text { a } 166 \text { participantes } \\
1 \text { ensaio } 453 \text { participantes (apenas mulheres) } \\
<20 \% \text { homens em cada ensaio, }<7 \% \text { no total dos } 5 \\
\text { ensaios }\end{array}$ \\
\hline Follow-up & $\begin{array}{l}1 \text { ensaio: } 60 \% \\
4 \text { ensaios: }>80 \%\end{array}$ & 5 ensaios: $>80 \%$ \\
\hline $\begin{array}{l}\text { Análise dos dados } \\
\text { com intenção de } \\
\text { tratar }\end{array}$ & $\begin{array}{l}2 \text { ensaios: sim } \\
3 \text { ensaios: não }\end{array}$ & $\begin{array}{l}3 \text { ensaios: sim } \\
2 \text { ensaios: não }\end{array}$ \\
\hline Outcome & $\begin{array}{l}4 \text { ensaios: bacteriúria e sintomas urinários } \\
1 \text { ensaio: apenas bacteriúria }\end{array}$ & $\begin{array}{l}4 \text { ensaios: bacteriúria e sintomas urinários } \\
1 \text { ensaio: apenas bacteriúria } \\
\text { ( } 3 \text { ensaios: leucocitúria) }\end{array}$ \\
\hline $\begin{array}{l}\text { Resultados (grupos } \\
\text { experimentais) }\end{array}$ & $\begin{array}{l}\text { Menor número de recorrências de ITU nos } 5 \\
\text { ensaios ( } p \text { entre }<0,0001 \text { e } 0,0025) \\
\text { Odds ratio global }=-2,28 \\
\text { resultados homogéneos entre os ensaios. }\end{array}$ & $\begin{array}{l}\text { Menor número de recorrências de infeções do trato } \\
\text { urinário nos } 5 \text { ensaios } \\
\text { diminuição da disúria em todos os ensaios - globalmente } \\
\text { menos } 9,4 \%(p<0,001) \\
\text { diminuição global da leucocitúria - } 13,3 \%(p<0,001) \text { - e } \\
\text { bacteriúria - } 6,2 \%(p<0,05) \text {. }\end{array}$ \\
\hline Nível de evidência & 2 & 1 \\
\hline
\end{tabular}




\section{Estudo de coorte}

A coorte prospetiva de Kim et a/ ${ }^{6}$ contemplou uma amostra de 34 participantes durante um episódio de ITU, com mais de dois episódios de ITU nos últimos seis meses, que foram tratados com antibioterapia. Após confirmação da resolução da infeção, realizaram profilaxia com uma cápsula diária de polissacarídeo de $E$. coli durante três meses e monitorizados por mais seis meses. Daqueles, 28 participantes não tiveram recorrência de ITU, e nos restantes a gravidade dos sintomas foi menor (Tabela 3 ).

\section{Metanálises}

A metanálise de Bauer et $a \Gamma$ integrou cinco estudos duplamente cegos. Os participantes dos estudos tinham antecedentes de ITU recorrente e apresentavam episódio de ITU no início do estudo; foram tratados com antibioterapia. Alguns participantes receberam polissacarídeo de E. coli diário e outros placebo, durante três meses; todos foram monitorizados durante mais três meses. Num dos estudos os participantes receberam o polissacarídeo durante ainda mais três meses - nos primeiros dez dias de cada mês - e monitorizados por mais três meses, completando um total de 12 meses.

A metanálise Naber et $a^{8}$ integrou cinco ensaios clínicos duplamente cegos, que apresentam metodologias semelhantes aos que integram a metanálise anterior.

Em todos os estudos verificou-se uma diminuição das recorrências no grupo experimental $(p<0,001)$ (Tabela 4).

\section{Guidelines}

As duas guidelines encontradas foram publicadas pela Sociedade Europeia de Urologia em $2009^{9}$ e em $2015^{10}$. A primeira, baseada em ensaios controlados duplamente cegos, defende que a OM 8930 reduziu de forma significativa as ITUs recorrentes comparativamente ao placebo; contudo, considera ser necessário mais estudos para corroborar a sua efetividade em relação à profilaxia antibiótica. A segunda defende que a evidência existente é suficiente e suporta a superioridade da OM 8930 face ao placebo, podendo, por isso, ser recomendada como imunoprofilaxia em utentes do sexo feminino com ITU recorrente não complicada. Salvaguardam que falta esclarecer a sua eficácia em outros grupos de doentes e a sua superioridade em relação à profilaxia antimicrobiana.

\section{DISCUSSÃO}

Atribuímos um nível de evidência 1 aos ensaios clínicos, pois são aleatorizados, apresentam uma metodologia sólida com um tamanho amostral consistente, follow-up superior ou igual a $80 \%$ e com outcomes orientados para a doença e para o doente. No ensaio clínico de Tammen et al, é ainda evidente o prolongamento do efeito profilático. Neste, a definição de bacteriúria na recorrência de ITU (104 microrganismos $/ \mathrm{mL}$ ) é diferente do valor admitido como critério de inclusão, contudo foi aplicado a ambos os grupos. No ensaio de Magasi et al, não foi definido o critério de ITU recorrente, mas todos os participantes apresentaram pelo menos um episódio de ITU ao início do ensaio clínico; assim, consideramos que o fato dos participantes terem feito mais dois episódios nos últimos 12 meses ou apenas um nos últimos seis meses não interfere na interpretação dos dados. Admitimos que os autores excluíram indivíduos com episódios recorrentes de pielonefrite para tal não ser confundidor de uma possível anomalia do trato urinário subjacente.

À coorte realizada por Kim et al, atribuímos o nível de evidência 1, pois apresenta uma metodologia consistente, com um follow-up superior a $80 \%$ e a duração da observação é o dobro da duração do tratamento, e tem outcomes orientados para o doente e para a doença. Apesar do número inicial de indivíduos do estudo ser relativamente pequeno (42 participantes), os autores referem ser superior ao necessário estimado (30). Os autores excluíram os dois casos em que a ITU recorreu durante o período de administração do polissacarídeo de E. coli, o que poderá aumentar os resultados pós-imunoprofilaxia; contudo, é plausível considerarmos que esta exclusão não alteraria o outcome.

À metanálise realizada por Bauer et al atribuímos o nível de evidência 2, uma vez que, apesar dos ensaios que a integram apresentarem uma metodologia sólida e o número total de participantes ser consistente, um dos ensaios apresenta um follow-up de apenas $60 \%$, diminuindo assim o número de indivíduos que fazem parte da análise estatística final; por esse facto e pelo número substancial de participantes excluídos em outros dois ensaios, Bauer et al consideraram que, em três dos ensaios primários, não se verificou análise dos dados com intenção de tratar. Aqui foi demonstrada uma redução de 2,28 vezes da probabilidade de um indivíduo que se submeteu à imunoprofilaxia com polissacarídeo de $E$. coli desenvolver uma recorrência da ITU (nos primeiros três meses após tratamento), o que traduz um impacto moderado-a-elevado da eficácia do seu uso.

À metanálise de Naber et al atribuímos nível de evidência 1, uma vez que os ensaios que a integram apresentam uma metodologia consistente, com um bom número

Tabela 4 - Síntese das duas guidelines selecionadas na pesquisa

\begin{tabular}{|c|c|c|}
\hline Referência & Cruz et a ${ }^{9}$ & Grabe et $a^{10}$ \\
\hline Resumo & $\begin{array}{l}\text { A vacina OM } 8930 \text { reduz de forma significativa as } \\
\text { infeções do trato urinário recorrentes. } \\
\text { Necessários mais estudos para comparar a sua } \\
\text { efetividade à da profilaxia antibiótica. }\end{array}$ & $\begin{array}{l}\text { A vacina OM } 8930 \text { pode ser recomendada em utentes do } \\
\text { sexo feminino com ITU recorrente não complicada. } \\
\text { Fundamental esclarecer a sua eficácia noutros grupos e em } \\
\text { comparação à profilaxia antimicrobiana. }\end{array}$ \\
\hline
\end{tabular}

ITU: infeção do trato urinário 
de participantes (superior a 1000), todos com um follow-up superior a $80 \%$ (um deles com o dobro de duração do tempo de exposição), e em três dos quais verificou-se análise dos dados com intenção de tratar. Dos estudos integrados desta metanálise, três apresentam participantes de ambos os sexos, sem que tenha havido análise por grupos (ou seja, ajuste à variável 'género'); contudo, os homens corresponderam a menos de $20 \%$ da população em cada um desses estudos e menos de $7 \%$ do total de participantes da metanálise. Os autores concluíram que, ao fim de três meses, o número de recorrências de ITU foi 35,7\% inferior no grupo experimental; também concluíram que nesse grupo houve menos 19,1\% de indivíduos sem qualquer recorrência em comparação com o placebo, também ao fim de três meses. Estes valores aumentam para $39,4 \%$ e $20,7 \%$, respetivamente, quando analisada a totalidade do período de estudo. Todos os ensaios clínicos da metanálise demonstraram diminuição do número de recorrências de ITU, da disúria, da bacteriúria e da leucocitúria (esta última somente nos três estudos que a caracterizaram), ainda que nem sempre de forma estatisticamente significativa individual.

Desta forma, a nossa revisão apresenta três estudos primários e dois secundários: dois ensaios clínicos randomizados, uma coorte prospetiva e uma metanálise aos quais foi atribuído nível de evidência 1, e uma outra metanálise à qual foi atribuído nível 2. Todos os estudos permitem concluir que a utilização do polissacarídeo de E. coli diminui o número de recorrências de ITU, de forma estatisticamente significativa. Assim, atribuímos força de recomendação $A$ à orientação do uso dessa vacina como imunoprofilaxia da ITU recorrente, baseada num corpo de

\section{REFERÊNCIAS}

1. Pereira S. Prevenção das infeções urinárias recorrentes. Urologia em Medicina Familiar. 2012;6:1-8.

2. Hooton TM, Gupta K. Recurrent urinary tract infection in women. Uptodate.com [Internet]. United States: Uptodate; atualizado 21 novembro 2016. [consultado 2016 abr 14]. Disponível em: https://www. uptodate.com/contents/recurrent-urinary-tract-infection-in-women.

3. Ebell MH, Siwek J, Weiss BD, Woolf SH, Susman J, Ewigman B, et al. Strength of Recommendation Taxonomy (SORT): a patient-centered approach to grading evidence in the medical literature. Am Fam Physician. 2004;69:549-57.

4. Tammen H, Buchholz G, Chichakli Y, Gabor M, Hess K, Hutzel WA, et al. Immunobiotherapy with Uro-Vaxom ${ }^{\circledR}$ in recurrent urinary tract infection. Br J Urol. 1990;65:6-9.

5. Magasi P, Pánovics J, Illés A, Nagy M. Uro-Vaxom ${ }^{\circledR}$ and the management of recurrent urinary tract infection in adults: a randomized multicenter double-blind trial. European Urology. 1994;26:137-40.

6. Kim KS, Kim J, Jeong IG, Paick J, Son H, Lim DJ, et al. A prospective evidência globalmente classificado por nós com nível 1 . Esta recomendação também é preconizada pelas duas guidelines encontradas.

Admitimos que a administração do polissacarídeo de $E$. coli durante três meses diminui o número de recidivas na ITU recorrente simples, com melhoria dos sintomas urinários e da bacteriúria, com impacto moderado/moderado-a-elevado.

\section{CONCLUSÃO}

Quanto à aplicabilidade na prática clínica, recomendamos a OM 8930 para a prevenção de ITUs de repetição não complicadas numa posologia de $6 \mathrm{mg} /$ dia durante três meses, após finalização do ciclo de antibioterapia relativo à infeção prévia. Contudo, consideramos fundamental a decisão partilhada, tendo em conta o custo/benefício do tratamento.

Julgamos essencial a realização de mais estudos para avaliar a relação custo/efetividade da vacina OM 8930 a médio e longo prazos e para estabelecer a sua eficácia noutros grupos de doentes, nomeadamente nas ITUs complicadas, e também em comparação com a profilaxia antimicrobiana.

\section{CONFLITOS DE INTERESSE}

Os autores declaram não ter nenhum conflito de interesse relativamente ao presente artigo.

\section{FONTES DE FINANCIAMENTO}

Não existem fontes externas de financiamento para a realização deste artigo.

multi-center trial of Escherichia coli extract for the prophylactic treatment of patients with chronically recurrent cystitis. J Korean Med Sci. 2010;25:435-9.

7. Bauer HW, Rahlfs VW, Lauener PA, Bleßmann G. Prevention of recurrent urinary tract infections with immuno-active E. coli fractions: a meta-analysis of five placebo-controlled doubleblind studies. Int $\mathrm{J}$ Antimicrob Agents. 2002;19:451-6.

8. Naber KG, Cho Y, Matsumoto T, Schaeffer AJ. Immunoactive prophylaxis of recurrent urinary tract infections: a meta-analysis. Int J Antimicrob Agents. 2009;33:111-9.

9. Cruz F, Dambros M, Naber KG, Bauer HW, Cozma G. Recurrent urinary tract infections: Uro-Vaxom ${ }^{\circledR}$, a new alternative. Eu Urol Suppl. 2009;8:762-8.

10. Grabe M, Bartoletti R, Johansen TE, Cai T, Çek M, Köves B, et al. Guidelines on Urological Infections. European Association of Urology; 2015. [consultado 2016 abr 26]. Disponível em: https://uroweb.org/wpcontent/uploads/19-Urological-infections_LR2.pdf. 